\title{
Resonance control of membrane reflectors with effective index engineering
}

\author{
Hongjun Yang, ${ }^{1}$ Santhad Chuwongin, ${ }^{1}$ Zexuan Qiang, ${ }^{1}$ Li Chen, ${ }^{1}$ Huiqing Pang, ${ }^{2}$ \\ Zhenqiang Ma, ${ }^{2, a)}$ and Weidong Zhou ${ }^{1, b)}$ \\ ${ }^{1}$ Department of Electrical Engineering, NanoFAB Center, University of Texas at Arlington, Arlington, \\ Texas 76019, USA \\ ${ }^{2}$ Department of Electrical and Computer Engineering, University of Wisconsin-Madison, Madison, \\ Wisconsin 53706, USA
}

(Received 21 April 2009; accepted 22 June 2009; published online 14 July 2009)

\begin{abstract}
We report here broadband membrane reflectors based on Fano resonances in patterned silicon nanomembranes. Resonance control of the reflectors was realized either by partially removing buried oxide layer underneath the device layer or by controlled $\mathrm{SiO}_{2}$ film deposition on top of the devices. Both blueshifts and redshifts were demonstrated with a turning range of $50 \mathrm{~nm}$ for a center wavelength of $1550 \mathrm{~nm}$. These results demonstrate practical postprocess means for Fano resonance engineering for both narrowband filters and ultracompact broadband reflectors. (C) 2009 American Institute of Physics. [DOI: 10.1063/1.3182801]
\end{abstract}

Surface-normal ultracompact narrowband optical filters and broadband reflectors are essential components for numerous device applications. They can be used in optical switches, modulators, lasers, sensors, and beam steering devices. One-dimensional subwavelength grating (SWG) structures have long been recognized for this purpose. Both narrowband filters and broadband mirrors ${ }^{1-4}$ based on SWG have been demonstrated. On the other hand, two-dimensional photonic crystal slab (2D PCS) structures hold great promises in realizing more compact photonic devices and they can be used for both in-plane and vertically coupled light guiding and manipulating. In this regard, Fano resonance can be explored for device applications. Based on Fano resonances, out-of-the-plane optical mode coupling in 2D PCS becomes feasible because the in-plane guided resonances above the lightline are strongly coupled to the out-of-the-plane radiation modes due to phase matching provided by the periodic lattice structure. ${ }^{5}$ In recent years, devices based on Fano resonances such as narrowband filters ${ }^{1,4}$ and broadband reflectors $^{6-8}$ have been reported. Recently, we also reported narrowband Fano filters and broadband membrane reflectors with patterned silicon nanomembranes (SiNM) transferred onto glass and flexible polyethylene terephthalate substrates, employing a low temperature wet-transfer process. ${ }^{10,11}$ The angular and polarization properties of Fano filters can also be tailored with the dispersion engineering, which is highly desirable for photonic integration applications. ${ }^{11,12}$ However, none of the previous works demonstrated resonance control which is needed in many practical applications.

Here we report the postfabrication resonance control for broadband membrane reflectors with precise control of cavity resonances, based on the effective index control of low index medium surrounding patterned SiNM device layers. The membrane reflector design was carried out based on three-dimensional finite difference time-domain simulations and rigorous coupled-wave analysis techniques. ${ }^{11,12}$ With the control of design parameters, broadband reflectors with various spectral bandwidths and different peak reflections can all be realized. The PCS structure was fabricated on silicon-on-

\footnotetext{
${ }^{a)}$ Electronic mail: mazq@engr.wisc.edu.

${ }^{b)}$ Electronic mail: wzhou@uta.edu.
}

insulator (SOI) wafers, using e-beam lithography and plasma dry-etching processes. ${ }^{11}$ The target wavelength is around $1550 \mathrm{~nm}$.

A membrane reflector fabricated on the SOI substrate is shown schematically in Fig. 1(a). It is also denoted as the "ASO" structure, following the sequence of the vertical confinement layers: air (top)-silicon-oxide (bottom). Shown in Figs. 1(b) and 1(c) are the cross-sectional view and top view of the scanning electron micrographs (SEMs) of the fabricated membrane reflectors on SOI substrates, respectively. The periodicity $(a)$ and air hole radius $(r)$ in this square lattice structure are 980 and $274 \mathrm{~nm}$, respectively. The thickness of the thin Si device layer is $365 \mathrm{~nm}$. High quality etching is essential in achieving the desired reflection perfor-
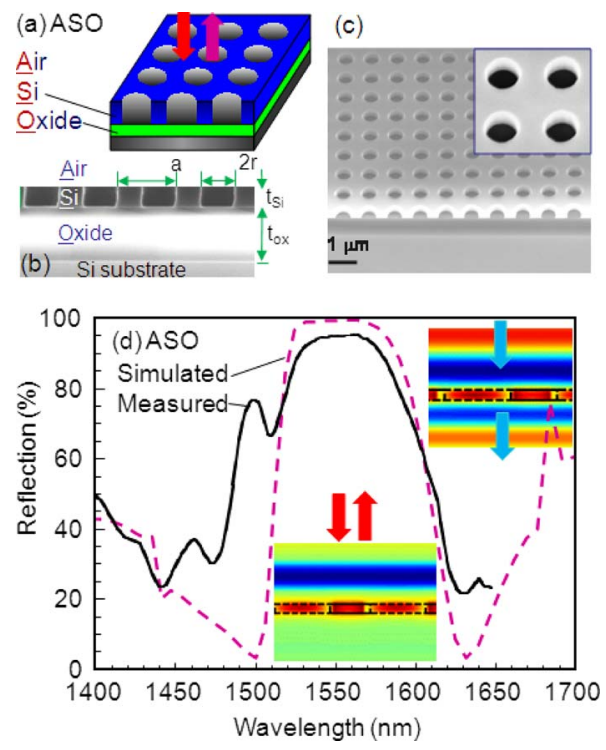

FIG. 1. (Color online) (a) Schematic of a membrane reflector with vertical ASO confinement configuration. (b) A cross-sectional SEM image of a membrane reflector on SOI, with key parameters labeled. (c) An angled, top-view SEM image of the membrane reflector, with a close-up view shown in the inset. (d) Measured and simulated reflection spectra for a membrane reflector with $a=980 \mathrm{~nm}, r / a=0.28, t_{\mathrm{Si}}=365 \mathrm{~nm}$, and $t_{\mathrm{ox}}$ $=1 \mu \mathrm{m}$. The simulated electrical field plots are also shown in the insets of (d) for both reflection band (bottom) and transmission band (top). 


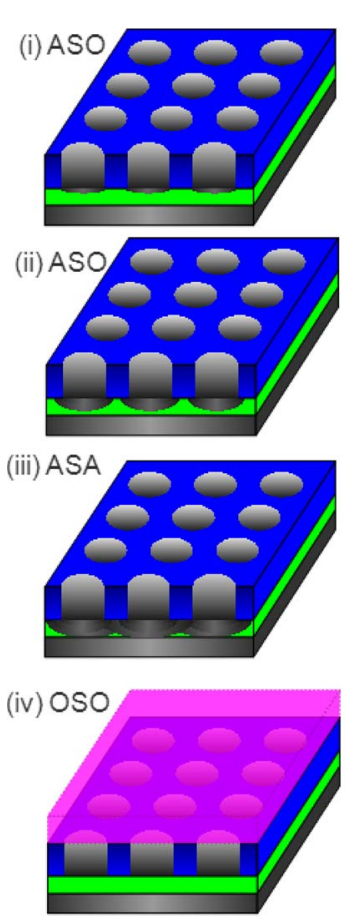

(a)
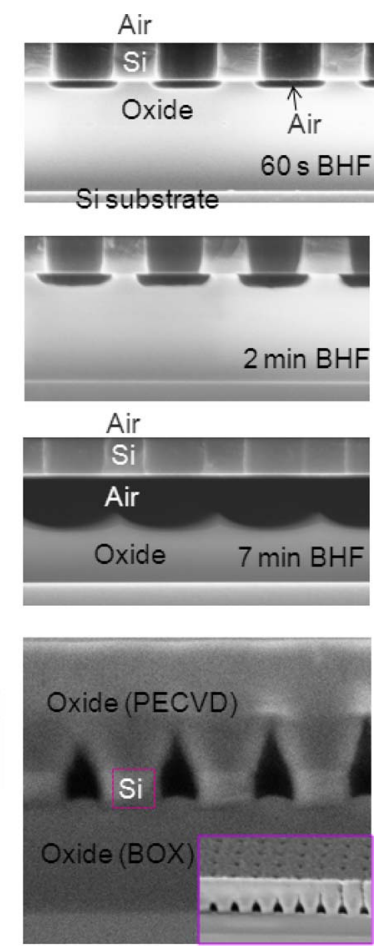

(b)
FIG. 2. (Color online) (a) Schematic and (b) cross-sectional SEM images of spectral trimming processes with controlled BHF etching time $(60 \mathrm{~s}, 2 \mathrm{~min}$, and $7 \mathrm{~min}$ ) of BOX layer for blueshift and controlled oxide deposition for redshift.

mance. Shown in Fig. 1(d) are the measured and simulated reflection spectra. Notice that some degradation of measured maximum reflection may be due to process imperfection. Theoretically, the maximum reflection can be $\sim 100 \%$. The simulated electrical field plots are also shown in the insets of Fig. 1(d) for both the reflection band (bottom) and the transmission band (top).

It is well known that photonic crystal and Fano resonance spectral properties can be controlled by varying design parameters, such as the lattice structure and material indices, including the medium surrounding the patterned photonic crystal layer. As a result, postprocess spectral tuning is feasible by controlling the effective indices of surrounding environment. In order to precisely trim the spectral locations, we report the following two approaches to precisely control the effective indices below and above the Si device layer: (1) to realize a spectral blueshift by reducing the effective index of material below the device with controlled partial etching of $\mathrm{SiO}_{2}$ buried oxide (BOX) layer and (2) to achieve a spectral redshift by increasing the effective index of material above the device with oxide deposition on top.

The processes for realizing blueshift are shown schematically in Fig. 2(a)(i)-(iii), along with the corresponding cross-sectional SEM images shown in Fig. 2(b). With controlled buffered hydrofluoric acid (BHF) etching times, the BOX layer is partially etched away, which results in the reduction in the effective index of the bottom oxide layer [Fig. 2(i) and (ii)]. When the BHF etching time is sufficiently long [e.g., $>7$ min, Fig. 2(iii)], the Si device layer is completely released (still supported at edges) with bottom layer effective index approaching the index of air ["ASA" stands for air (top)-silicon-air (bottom)].
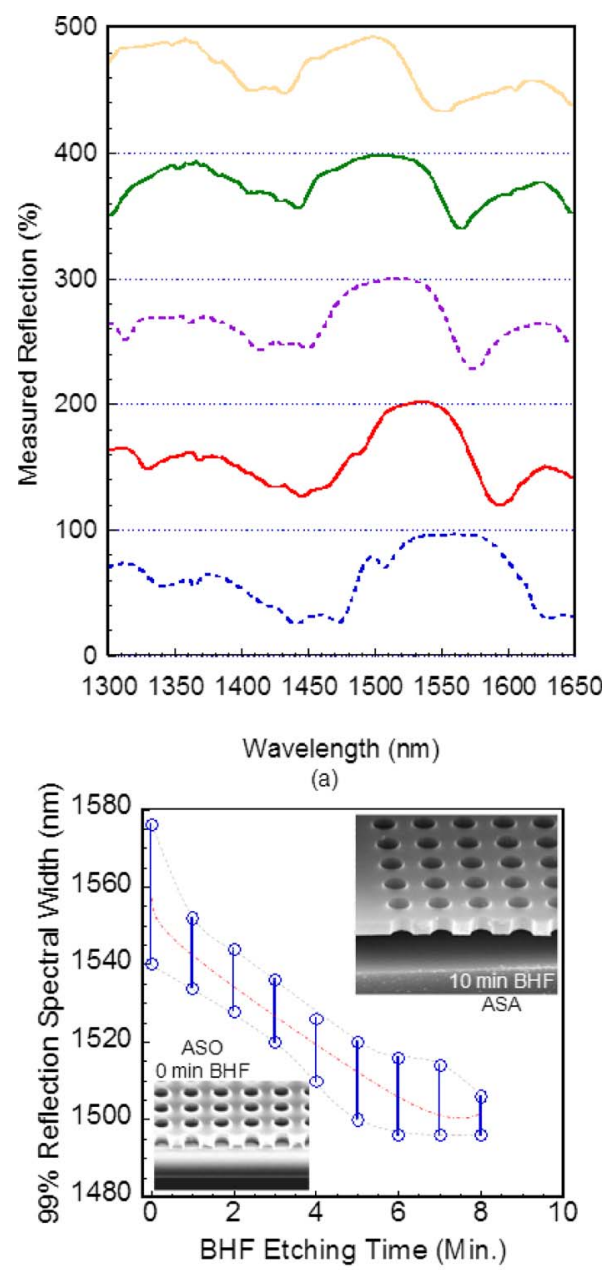

(b)

FIG. 3. (Color online) Measured reflection characteristics. (a) Reflection spectra for membrane reflectors with different BHF etching times (from 0 to $8 \mathrm{~min}$ ). The reflection spectra are shifted up by multiples of 100 for different etching times to provide a better view of the spectra. (b) $99 \%$ reflection spectra bands for different BHF etching times. Shown in the insets are the SEM images for Fano filters with ASO (0 min BHF etching time) and ASA (10 min BHF etching time) configurations.

The measured broadband reflection results are shown in Fig. 3 for different BHF etching times. The measured spectra are shown in Fig. 3(a), where the spectra curves are shifted by multiples of 100 to have a better view for comparison. The reflection spectra bandwidths with $99 \%$ reflection are summarized in Fig. 3(b). The center wavelengths [red dotted line in Fig. 3(b)] shifted almost linearly for etching time less than $7 \mathrm{~min}$, when the top portion of BOX oxide layer are completed removed [Fig. 2(b)iii]. Beyond $7 \mathrm{~min}$, further etching has little impact on the center wavelength of the reflection bands, simply because the effective index underneath the device has no more changes (air). The results indicate that the Fano resonance shift is only related to the effective index of very thin layers $(\sim 200 \mathrm{~nm})$ above and below the patterned SiNM layer where field interaction is strong [inset of Fig. 1(d)]. As seen in Fig. 3, a total of $53 \mathrm{~nm}$ blueshift was obtained for a total etching time of $6 \mathrm{~min}$. It is worth mentioning that similar trends can be found for the reflection spectra bands at different reflection levels (e.g., $99.9 \%, 95 \%$, etc.).

Similarly, redshift via spectral trimming can also be realized with the addition of an oxide layer surrounding the 


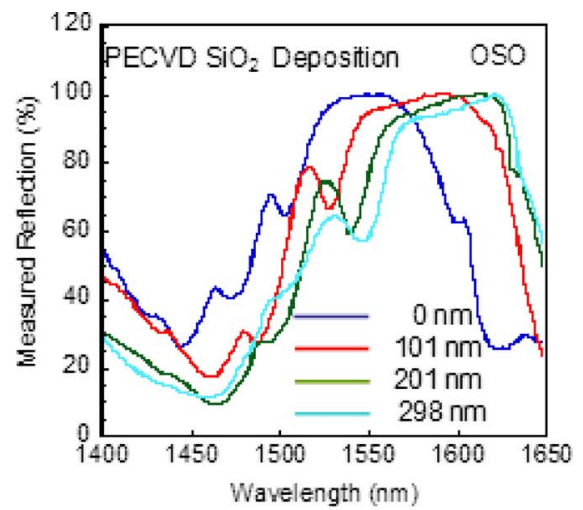

(a)

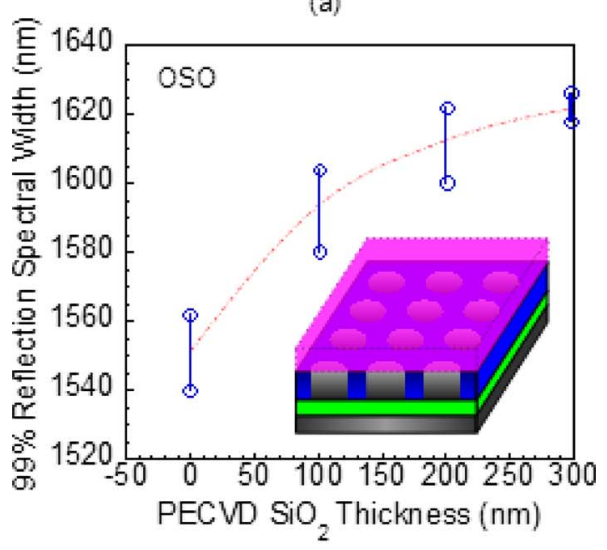

(b)

FIG. 4. (Color online) (a) Measured reflection spectra for different $\mathrm{SiO}_{2}$ thicknesses deposited on top of the Si device layer (from 0 to $298 \mathrm{~nm}$ ) and (b) $99 \%$ reflection spectral widths for different PECVD oxide thicknesses.

patterned Si device layers with different thicknesses. This can be accomplished with either plasma-enhanced chemical vapor deposition (PECVD) of oxides on the top of Si device layer, or with spin-on-glass (SOG) backfilling of the air regions above, below and in the air holes. The oxide deposition process is schematically shown in Fig. 2(iv), where a $1 \mu \mathrm{m}$ thick PECVD oxide is deposited on the patterned Si layer. Notice that the air hole is partially filled with oxide deposited during the initial phase of deposition process. This is typical where PECVD oxide cannot fill nanoscale air holes effectively. A more conformal process can be introduced to fill the air holes more effectively by the SOG coating process. The oxide (top)-silicon-oxide (bottom) structure is denoted as "OSO."

The measured reflection results are shown in Fig. 4 for different PECVD oxide thicknesses. Notice a saturation behavior in resonance shift is observed for the oxide layer beyond $150 \mathrm{~nm}$. For the initial oxide deposition thickness from 0 to $100 \mathrm{~nm}$, the resonance can shift $45 \mathrm{~nm}$ toward longer wavelengths at a rate of $0.45 \mathrm{~nm}$ (wavelength)/nm (oxide).

Finally, we demonstrated spectral trimming with SOG are on the same SOI sample, as shown in Fig. 5. Beginning with an as-fabricated ASO structure, which has a peak reflection at $1551 \mathrm{~nm}$, the spectral peak first shifted to $1500 \mathrm{~nm}$ after controlled BOX layer removal [ASA shown in Fig.

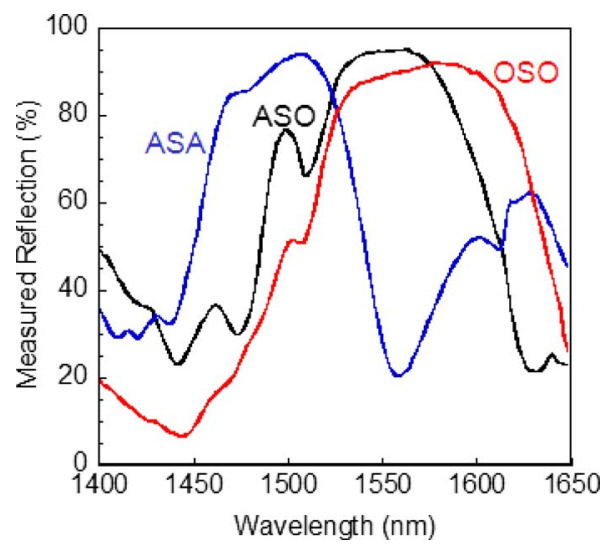

FIG. 5. (Color online) Measured reflection spectra for membrane reflectors with three configurations: ASO as fabricated, ASA realized with a 4 min BHF etching from the ASO structure, and OSO with SOG backfill of the ASA structures.

2(iii)]. The peak reflection then shifted back to $1580 \mathrm{~nm}$ after the sample was back-filled with spin-coated SOG solution [also denoted as OSO, as shown in Fig. 2(iv)]. The SOG solution used here is the Honeywell Accuglass 211 with thickness of $\sim 200 \mathrm{~nm}$.

Fano resonance broadband reflectors are realized with the controlled spectral locations based on the postfabrication spectral processes. Spectral trimming was accomplished by partially removing the BOX layer underneath the device layer or by controlled $\mathrm{SiO}_{2}$ film deposition on the top. Resonance tuning was demonstrated with a turning range over $50 \mathrm{~nm}$ for both blueshifts and redshifts, making it a practical postprocess design tool for Fano resonance trimming for both narrowband filters and ultracompact broadband reflectors.

This work was supported in part by the U.S. AFOSR MURI program under Grant No. FA9550-08-1-0337 and in part by U.S. DARPA under Grant No. HR0011-08-1-0058.

${ }^{1}$ R. Magnusson and S. S. Wang, Appl. Phys. Lett. 61, 1022 (1992).

${ }^{2}$ M. C. Y. Huang, Y. Zhou, and C. J. Chang-Hasnain, Nat. Photonics 1, 119 (2007).

${ }^{3}$ S. Boutami, B. B. Bakir, P. Regreny, J. L. Leclercq, and P. Viktorovitch, Electron. Lett. 43, 282 (2007).

${ }^{4}$ A. Rosenberg, M. Carter, J. Casey, M. Kim, R. Holm, R. Henry, C. Eddy, V. Shamamian, K. Bussmann, S. Shi, and D. W. Prather, Opt. Express 13, 6564 (2005).

${ }^{5}$ S. Fan and J. D. Joannopoulos, Phys. Rev. B 65, 235112 (2002).

${ }^{6}$ W. Suh and S. Fan, Appl. Phys. Lett. 84, 4905 (2004).

${ }^{7}$ S. Boutami, B. B. Bakir, H. Hattori, X. Letartre, J. L. Leclercq, P. RojoRomeo, M. Garrigues, C. Seassal, and P. Viktorovitch, IEEE Photonics Technol. Lett. 18, 835 (2006).

${ }^{8}$ V. Lousse, W. Suh, O. Kilic, S. Kim, O. Solgaard, and S. Fan, Opt. Express 12, 1575 (2004).

${ }^{9}$ H. Yang, Z. Qiang, H. Pang, Z. Ma, and W. D. Zhou, Electron. Lett. 44 858 (2008).

${ }^{10}$ H. C. Yuan, G. K. Celler, and Z. Ma, J. Appl. Phys. 102, 034501 (2007)

${ }^{11}$ Z. Qiang, H. Yang, L. Chen, H. Pang, Z. Ma, and W. D. Zhou, Appl. Phys. Lett. 93, 061106 (2008).

${ }^{12}$ L. Chen, Z. Qiang, H. Yang, H. Pang, Z. Ma, and W. D. Zhou, Opt Express 17, 8396 (2009). 